Journal of Case Reports 2020;10(1):12-14

\title{
Iatrogenic Enterovesical Fistula
}

\author{
Saba Feraz, Sultan Ali, Neelam Jain, Urvashi Priya Sinha \\ Department of Radiology, Discovery Diagnostics, Jamshedpur, Jharkhand, India.
}

\begin{abstract}
Corresponding Author:
Dr Saba Feraz

Email: drsabaferaz@gmail.com

This is an Open Access article distributed under the terms of the Creative Commons Attribution License (creativecommons.org/ licenses/by/3.0).
\end{abstract}

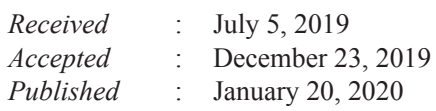

\begin{abstract}
Background: Enterovesical fistula is an abnormal communication between the gastrointestinal tract and urinary bladder. It can be due to varied etiologies including benign, malignant, trauma or iatrogenic. Cross-sectional imaging is an excellent method for detection, type, location, and etiology of fistula. Case Report: We hereby present a rare case of iatrogenic ileo-vesical fistula in a middle aged female after cystoscopic removal of urinary bladder calculus in untrained hands. Conclusion: Radiologists should be aware of this entity and keep iatrogenic injury as a possible cause of the fistula in presence of previous surgical history and if no other underlying obvious cause is present.
\end{abstract}

Keywords: Cystoscopy, Dysuria, Intestinal Fistula, Urinary Bladder Fistula, Urography.

\section{Introduction}

Entero-vesical fistula is an abnormal communication between small bowel loops and urinary bladder [1]. They present with suprapubic pain, recurrent polymicrobial urinary tract infections, pneumaturia or fecaluria. Cross-sectional imaging is an excellent method for detection, type, location, and etiology of fistula. We present here a case of iatrogenic ileo-vesical fistula after cystoscopic removal of urinary bladder calculus.

\section{Case Report}

A middle-aged female presented with complaints of suprapubic pain, dysuria and fecaluria for 5-6 months. On examination, the vitals of the patient were stable. Systemic and abdominal examinations were within normal limits except for mild tenderness in the supra-pubic region. Furthermore, routine blood investigations were normal, however, urine routine and microscopy revealed sediments with mild infection. Ultrasound (USG) abdomen [Fig.1] showed multiple intra-luminal echogenic floating particles without posterior shadowing in the urinary bladder. X-ray KUB was also advised which was negative for any radio-opaque shadow. Further CT urography [Fig.2,3] with intravenous iodine contrast showed air within the bladder and abnormal close approximation between small bowel loop and bladder with indistinct intervening fat plane and focal adjoining bladder wall thickening. No retrograde passage of excreted contrast from the bladder into bowel loops was seen. On persistent inquiry, the patient gave a history of previous cystoscopic removal of urinary bladder calculus by a surgeon. Diagnostic cystoscopy confirmed

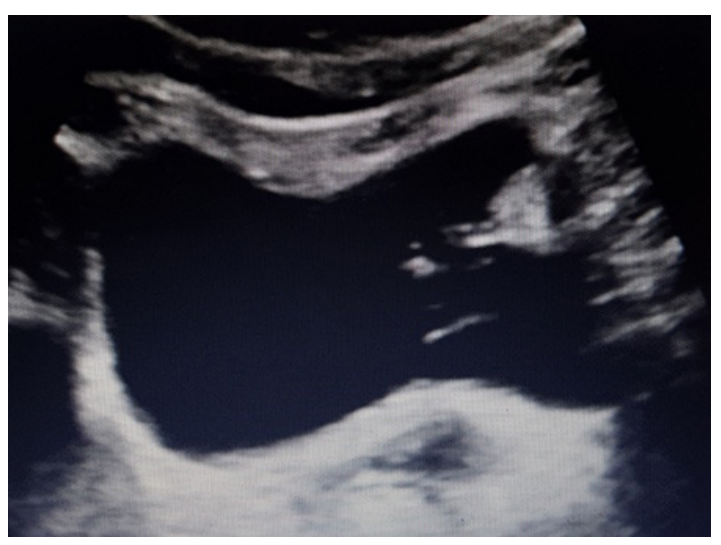

Fig.1: Transabdominal USG: floating echogenic intraluminal particles in urinary bladder. 
the presence of a small opening in the urinary bladder wall with intra-luminal fecal matters. This corroborated the possibility of enterovesical fistula, most likely due to injury during previous cystoscopic removal of urinary bladder calculus. The patient was advised surgery which she refused and preferred conservative management.

\section{Discussion}

Enterovesical fistula is an abnormal communication between the gastrointestinal tract and urinary bladder. Various etiologies of these fistulas include inflammatory bowel disease, advanced intestinal or urinary malignancies, radiotherapy, pelvic surgery, trauma or iatrogenic injuries [2-5]. Depending on the bowel segment involved, enterovesical fistula can be divided into ileo-vesical, appendico-vesical, colo-vesical or recto-vesical [4,5]. Ileovesical fistulas are most commonly a complication of Crohn's disease. Colovesical fistula is commonly seen in the setting of diverticular disease or colon cancer [5] while rectovesical fistula is usually due to trauma or neoplasm [1-3].

Clinical manifestations of entrovesical fistula include fecaluria, pneumaturia, recurrent UTIs, abdominal pain and malodourous urine [5]. Diagnostic workup includes poppy seed or charcoal test, cystography, barium studies, ultrasonography, and various cross-sectional imaging. Poppy seed $[5,6]$ or charcoal test involves the detection of orally consumed poppy seeds or charcoal (which remain undigested in gastrointestinal tract) in a centrifuged sample of urine. However, it does not provide any information regarding the location or etiology of the fistula [5].

Trans-abdominal ultrasonography can show floating intra-luminal debris (fecal particles) in the urinary bladder and abnormal approximation between a bowel loop and urinary bladder with indistinct intervening wall separation. Ultrasonography can sometimes reveal an echogenic "beak sign" connecting the peristaltic

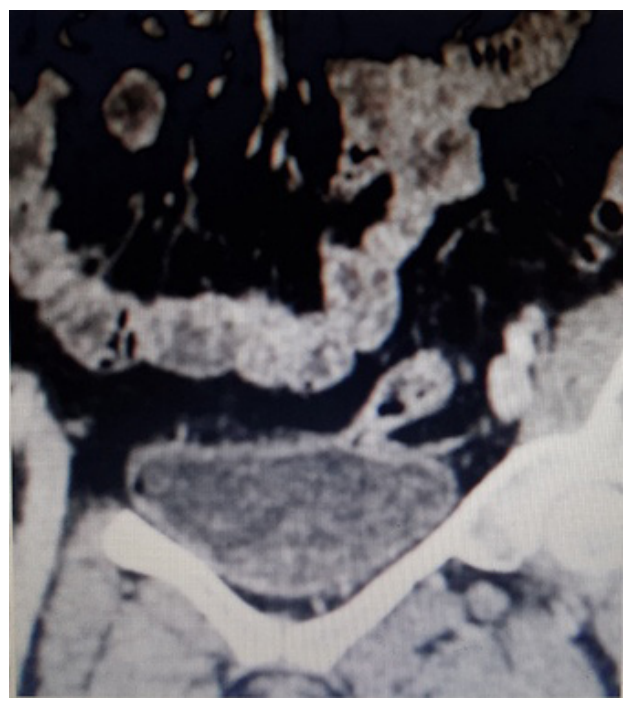

Fig.2: $C T$ urography sagittal view showing communication between ileal loop and urinary bladder with adjoining urinary bladder wall thickening.

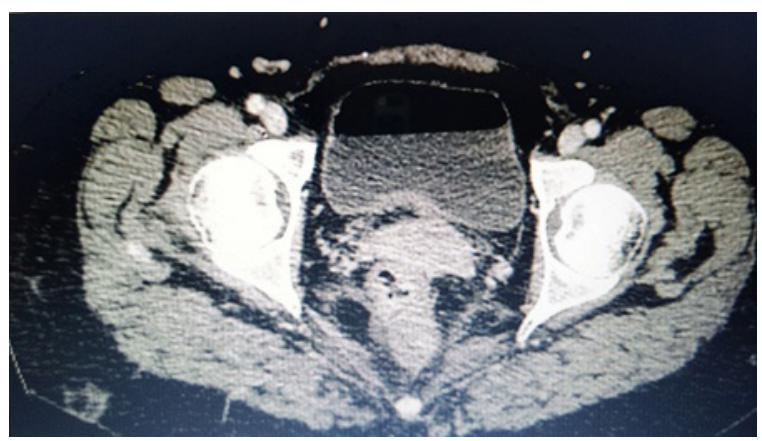

Fig.3: CT urography axial view showing air within the urinary bladder.

bowel lumen and the urinary bladder, after abdominal compression [5,7]. Trans-rectal and trans-vaginal ultrasonography can increase the chances of detection of fistula. However, it is highly dependent on the experience of the operator.

Plain abdominal X-ray and barium studies are usually inconclusive [5]. However, rarely barium studies can demonstrate the fistulous tract and also can reveal associated bowel pathology like in the case of inflammatory bowel disease or bowel malignancy. After barium GI study, barium can also be detected by radiography of a centrifuged sample of urine. Cystography can give 
direct or indirect evidence of enterovesical fistula like the direct visualization of the fistulous tract or indirectly by contrast opacification of bowel loops. Cross-sectional imaging (CT/ MRI) is the modality of choice as it can demonstrate the location and etiology of fistulous tract and also the extent of underlying disease $[5,8]$. Findings suggestive of an abnormal communication between bowel and urinary bladder are air within the bladder (with no prior history of bladder instrumentation or active urinary tract infection with a gas-forming organism), oral contrast medium within the bladder (in non IV contrast-enhanced scans) or focal eccentric bowel wall thickening [8]. MRI having excellent soft-tissue resolution can exactly delineate the fistulous tract and its complexity whether single or multiple tracts without the need of intra-luminal contrast. It is the investigation of choice for colo-vesical fistula with high specificity and sensitivity reaching up to 100 percent $[5,9,10]$. Cystoscopy can help in direct visualization of the fistulous opening. However, the detection rate is low and mostly non-specific finding of fistula like wall edema is seen [11]. Colonoscopy can be done in cases of colo-vesical fistula for assessment of underlying colonic pathology with tissue sampling. However, these procedures are invasive.

Management can be conservative or surgical depending on the symptoms of the patient and underlying disease. Conservative treatment includes bowel rest, total parenteral nutrition and antibiotics for UTIs and medication to suppress the activity of the underlying disease. Surgical management can be endoscopic, laparoscopic or open depending upon the complexity of fistula, part of bowel involved and underlying pathology [5].

\section{Conclusion}

Entero-vesical fistula can be a complication of diagnostic or therapeutic cystoscopy in untrained hands. Cross-sectional imaging like CT and MRI are the investigation of choice. Radiologists must be well acquainted with the radiological findings and should be able to correlate the findings with the symptomatology of the patient for making the correct diagnosis.

Contributors: SF: manuscript writing, literature review and imaging; SA, NJ: critical inputs into the manuscript and imaging; UPS: literature review, discussion and imaging. SF will act as a study guarantor. All authors approved the final version of this manuscript and are responsible for all aspects of the study.

Funding: None; Competing interests: None stated.

\section{References}

1. Li S, Chen Z, Zhang Q, Huang C, Wang Z, Du S. Four cases of enterovesical fistula and the importance of CT in the diagnosis. BJR Case Rep. 2017;2:20150124.

2. Kavanagh D, Neary P, Dodd JD, Sheahan KM, O'Donoghue D, Hyland JM. Diagnosis and treatment of enterovesical fistulae. Colorectal Dis. 2005;7:286-291.

3. Chen WS, Hsieh JH, Jiang JK, Lin JK, Hsu H, Lin TC. Enterovesical fistula: 10 years of experience. Chin Med J 1997;59:283-288.

4. Gross M, Peng B. Appendico-vesical fistula. J Urol.1969;102:697-698.

5. Golabek T, Szymanska A, Szopinski T, Bukowczan J, Furmanek M, Powroznik J, et al. Enterovesical fistulae: etiology, imaging, and management. Gastroentrol Res Tract. 2013;617967.

6. Kwon EO, Armenakas NA, Scharf SC, Panagopoulos G, Fracchia JA. The poppy seed test for colovesical fistula. Big bang, little bucks. J Urol. 2008;179:1425-1427.

7. Sutijono D. Point-of-care sonographic diagnosis of an enterovesical fistula. J Ultrasound Med. 2013;32:883885.

8. Goldman SM, Fishman EK, Gatewood OMB. CT in the diagnosis of enterovesical fistulae. AJR Am J Roentgenol. 1985;144:1229-1233.

9. Tang YZ, Booth TC, Swallow D, Shahabuddin K, Thomas M, Hanbury $\mathrm{D}$, et al. Imaging features of colovesical fistulae on MRI. Br J Radiol. 2012;85:13711375.

10. Amendola MA, Agha FP, Dent TL. Detection of occult colovesical fistula by the Bourne test. AJR Am J Roentgenol. 1984;142:715-718.

11. Melchior S, Cudovic D, Jones J, Thomas C, Gillitzer $\mathrm{R}$, Thüroff J. Diagnosis and surgical management of colovesical fistulas due to sigmoid diverticulitis. J Urol. 2009;182:978-982. 